\title{
Identification of the propagation mode of a solar wind wave associated with Pc5 pulsations in the magnetosphere
}

\author{
A. D. M. Walker and J. A. E. Stephenson \\ School of Chemistry and Physics, University of KwaZulu-Natal, Durban, South Africa \\ Correspondence to: A. D. M. Walker (walkerd@ukzn.ac.za)
}

Received: 15 August 2014 - Revised: 5 September 2014 - Accepted: 11 September 2014 - Published: 10 October 2014

\begin{abstract}
A case study of a magnetohydrodynamic (MHD) wave in the solar wind that is strongly correlated with a magnetospheric field line resonance observed by the SuperDARN (Super Dual Auroral Radar Network) radar at Sanae, Antarctica is presented. The data from the ACE (Advanced Composition Explorer) satellite at the solar libration point are analysed. The data time series are bandpass filtered at the pulsation frequency and the analytic signal deduced. From these data the partition of energy between the field components is computed. It is shown that energy is equally partitioned between the kinetic energy and transverse magnetic potential energy densities. The energy flux vector is closely aligned with the background magnetic field. The transverse magnetic and velocity components are in antiphase. This is the first identification of the triggering wave as a transverse Alfvén wave which originates upstream from the space craft and is propagated to the magnetosphere to trigger the pulsation.
\end{abstract}

Keywords. Magnetospheric physics (MHD waves and instabilities)

\section{Introduction}

In previous work (Stephenson and Walker, 2002, 2010), we showed strong correlation between field line resonances observed by the Sanae SuperDARN (Super Dual Auroral Radar Network) radar in Antarctica (Walker, 2002) and oscillations in the solar wind at the same frequency. Other authors have obtained similar results (see references cited by Stephenson and Walker, 2010). The modelling studies of Ghosh et al. (2009) show how such coherent waves can coexist with turbulence. Most studies of such waves have focused on spectral analysis. We are not aware of any studies that attempt to identify the waves by a detailed investigation of the relationships between their field components.

In this paper we introduce a new analysis technique for such waves and apply it to a case study of a solar wind oscillation that has been correlated with a field line resonance. We believe that this is the first study presenting unambiguous evidence that a transverse Alfvén wave, propagated parallel to the magnetic field in the solar wind rest frame and originating from a region upstream of the solar libration point, excited a field line resonance observed within the magnetosphere.

\section{The event of 7 June 2000}

Stephenson and Walker (2010) have previously reported on a number of solar wind events that are correlated with radar field line resonances. One of these was the event of 7 June 2000. Figure 1 shows the magnitude-squared coherence of ACE $V_{x}$ and Sanae Doppler velocity at the resonance, delayed by $57 \mathrm{~min}$, the time for the signal to propagate from spacecraft to ionosphere. The peak at $2.1 \mathrm{mHz}$ is significant at the $97 \%$ confidence level. There was also phase coherence between the signals, and there was correlation with data from the Wind satellite, located elsewhere in the solar wind. Similar behaviour was seen in several other events. The evidence strongly indicated that the solar wind oscillation was triggering the pulsation at the same frequency.

In this paper we focus attention on the nature of the $2.1 \mathrm{mHz}$ solar wind oscillations at the solar libration point using ACE data. 


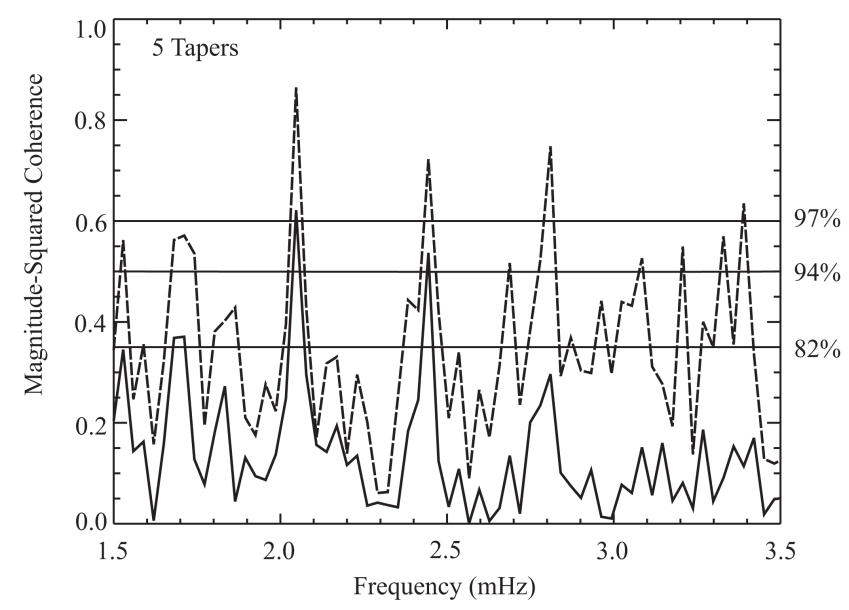

Figure 1. Magnitude-squared coherence (full line) between ACE $V_{x}$ and Sanae Doppler velocity in beam 4 at AACGM latitude $-66.4^{\circ} \mathrm{S}$ for the period from 09:50 to 19:00 UT on 7 June 2000. The horizontal lines show the cumulative distribution function estimates of the confidence level. The dashed line is drawn one standard deviation above the coherence curve. From Stephenson and Walker (2010).

\section{Some theoretical results}

Near $\beta=1$, three magnetohydromagnetic waves can exist; the fast and slow magnetosonic waves and the transverse Alfvén wave (Walker, 2005, chapter 7). The magnetosonic wave disturbances have important contributions from the plasma and magnetic field pressure: the transverse Alfvén wave is incompressible. The fast wave is not highly anisotropic, but energy in the slow wave is propagated approximately along the magnetic field. Alfvén wave energy is propagated exactly along the magnetic field for all wave normal directions.

The transverse Alfvén wave is incompressible with $p=0$ and is polarized with $\boldsymbol{b}$ and $\boldsymbol{v}$ in antiphase and perpendicular to the plane containing $\boldsymbol{B}$ and $\boldsymbol{k}$. The magnetosonic waves are compressible with non-zero $p$ and a parallel component of $\boldsymbol{b}$. The perturbations $\boldsymbol{b}$ and $\boldsymbol{v}$ lie in the plane containing $\boldsymbol{B}$ and $\boldsymbol{k}$.

In a stationary medium the wave energy density is

$U=\frac{1}{2} \rho_{0} v^{2}+\frac{1}{2} b^{2} / \mu_{0}+\frac{1}{2} p^{2} / \gamma P$

and the wave flux vector in the plasma rest frame is

$\boldsymbol{\Pi}_{0}=\left\{p+\frac{\boldsymbol{B} \cdot \boldsymbol{b}}{\mu_{0}}\right\} \boldsymbol{v}-\frac{\boldsymbol{B}(\boldsymbol{b} \cdot \boldsymbol{v})}{\mu_{0}}$,

and it can be shown that the flux vector represents the energy density being transported at the group velocity $V_{\mathrm{G}}$ so that

$\Pi_{0}=U V_{\mathrm{G}}$

(e.g. Walker, 2005).
In the solar wind the wave flux is

$\Pi=\Pi_{0}+U \boldsymbol{V}$.

$V$ is large enough for the second term to dominate. There is equipartition of energy between the kinetic energy density and potential energy density in the wave (Walker, 2014) so that, in general,

$\frac{1}{2} \rho_{0} v^{2}=\frac{1}{2} b^{2} / \mu_{0}+\frac{1}{2} p^{2} / \gamma P$.

In the case of the transverse Alfvén wave, $p=0$.

\section{Analysis of data for the event of 7 June 2000}

The data set consists of time series of solar wind magnetic field $\boldsymbol{B}$, velocity $\boldsymbol{V}$, proton number density $N_{H}$, and proton temperature $T_{H}$ measured at ACE. From $N_{H}$ and $T_{H}$ the pressure and mass density of the hydrogen plasma can be deduced. All data have been interpolated and resampled to give a common sampling rate of $64 \mathrm{~s}$, the same as that of the radar. The time series are $18 \mathrm{~h}$ long, starting at $01: 31$ on 7 June. All Fourier analysis is done using the multiple-taper method (MTM) (Thomson, 1982). This allows objective error analyses. The multiple tapers do not sacrifice data as does a single window and the method provides the best trade-off between variance and bias.

The background is estimated by taking a $58.7 \mathrm{~min}$ ( $55 \mathrm{sam}$ ples) boxcar average of the data. The periods of the disturbances of interest are about $8 \mathrm{~min}$. Throughout the period $\beta>1$, the Alfvén speed is close to $60 \mathrm{~km} \mathrm{~s}^{-1}$ and the sound speed varies from 40 to $60 \mathrm{~km} \mathrm{~s}^{-1}$. These speeds are small compared with the typical solar wind speed of about $400 \mathrm{~km} \mathrm{~s}^{-1}$.

The fields show perturbations on all timescales. There are two major peaks near 2.1 and $2.5 \mathrm{mHz}$, where there was strong correlation with the field line resonance event observed by the radar. Throughout the $18 \mathrm{~h}$ period of observation there is ULF (ultra low frequency) activity. Much of this is noise or weak turbulence.

We analyse the disturbance at the $2.1 \mathrm{mHz}$ peak. The mass density time series is found from $N_{H}$ using the proton mass. This is likely to be an underestimate as it ignores the possible presence of a small population of helium ions. The proton pressure is estimated from $T_{H}$, and this is assumed to be half the total pressure, the balance arising from electrons. The spectra of the time series for $P, \rho$, and the cartesian components of $B$ and $V$ have been bandpass filtered with a filter of width $0.168 \mathrm{mHz}$ centred on the $2.1 \mathrm{mHz}$ peak. The analytic signal in the time domain, found from complex demodulation, has then been calculated for each wave variable as described by Stephenson and Walker (2010).

The analytic signal is a complex time series whose real part is the actual filtered data series. For a quasi-sinusoidal signal, its amplitude is the instantaneous amplitude of the 


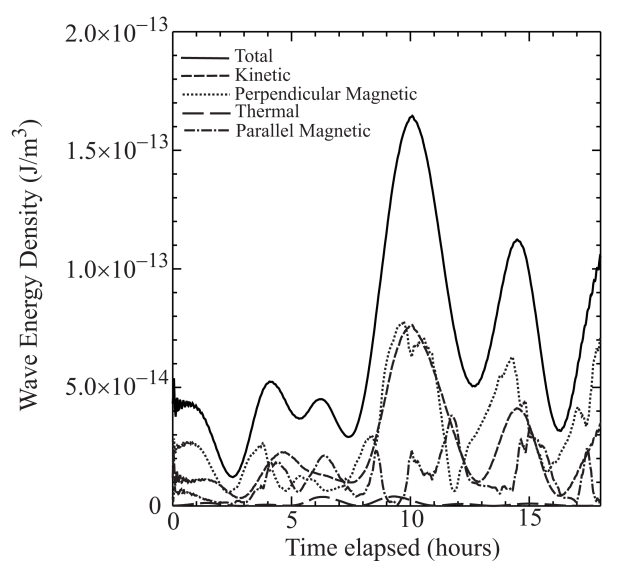

Figure 2. Partition of wave energy density. The wave energy density in the $2.1 \mathrm{mHz}$ band is shown by the full curve and the contributions from each field component by the broken curves. Time is measured from 01:31 on 7 June 2000.

wave and its phase is the instantaneous phase. We can use the amplitude to calculate the contribution of each field component to the internal energy of the wave. It is convenient to use the components of the wave magnetic field perturbation and velocity perpendicular and parallel to the background magnetic field $B$ so

$b_{||}=\boldsymbol{b} \cdot \boldsymbol{B} / B ; \quad b_{\perp}=b-b_{||} \boldsymbol{B} / B$,

with similar expressions for $v$. Then Eq. (1) may be written

$U=\frac{1}{2} \rho_{0} v_{\|}^{2}+\frac{1}{2} \rho_{0} v_{\perp}^{2}+\frac{1}{2} b_{\|}^{2} / \mu_{0}+\frac{1}{2} b_{\perp}^{2} / \mu_{0}+\frac{1}{2} p^{2} / \gamma P$.

Figure 2 shows the wave energy density as well as each contribution to its magnitude. The rms amplitudes of each component are used. It is clear that there is a large rise in wave activity during the period $8-12 \mathrm{~h}$ after the start of the event. There is a second peak in activity between 13 and $16 \mathrm{~h}$. First, consider the period from 0 to $8 \mathrm{~h}$. If we examine the contributions to the energy density during this period they all have the same order of magnitude except for the thermal pressure, which is negligible. Each varies independently with time. We conclude that during this period the wave activity was simply ULF noise and the value of $U$ is a measure of the noise level. If we now examine the peak between 8 and $12 \mathrm{~h}$, we see that it arises entirely from an increase in the energy associated with the velocity and magnetic field perturbations transverse to the background magnetic field. Further, the peaks in these two quantities are close to identical while the other contributions to the total wave energy continue with their noise-like behaviour. This behaviour is less clear during the second peak in wave activity between 13 and $16 \mathrm{~h}$. This peak has a smaller signal-to-noise ratio. The major contributions to the energy density still come from the transverse perturbations but the equipartition of energy is not clear.

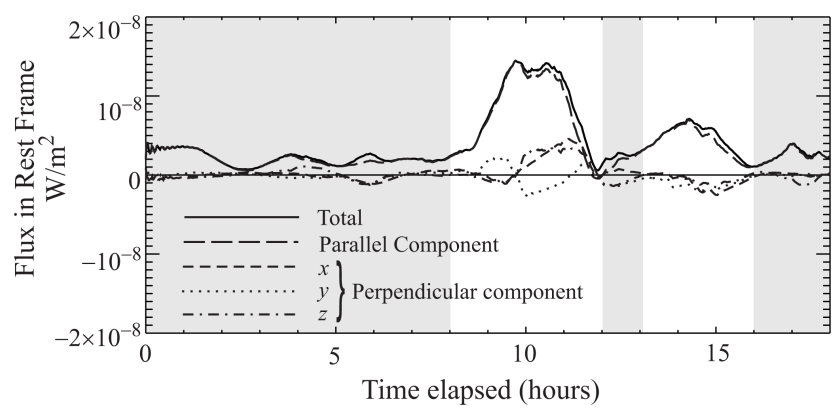

Figure 3. Magnitude of wave flux vector in rest frame of solar wind. Time is measured from 01:31 on 7 June 2000. The unshaded portions of the diagram indicate the peaks in wave energy density.

We now transfer our attention to the energy flux in the local solar wind rest frame. The data are transformed into a local coordinate system $\left\{x^{\prime}, y^{\prime}, z^{\prime}\right\}$ that varies slowly in time. In this system the $z^{\prime}$ axis is aligned with the magnetic field, the $x^{\prime}$ axis is perpendicular to this in the plane defined by the GSM $x$ axis, and the $y^{\prime}$ axis completes the right-hand set. Let $(\hat{\boldsymbol{x}}, \hat{\boldsymbol{y}}, \hat{\boldsymbol{z}})$ and $\left(\hat{\boldsymbol{x}}^{\prime}, \hat{\boldsymbol{y}}^{\prime}, \hat{\boldsymbol{z}}^{\prime}\right)$ be unit vectors in the direction of the GSM and local axes respectively. Then

$$
\begin{aligned}
\hat{\boldsymbol{z}}^{\prime} & =\boldsymbol{B}_{0} / B_{0}, \\
\hat{\boldsymbol{y}}^{\prime} & =\boldsymbol{B}_{0} \times \hat{\boldsymbol{x}} / B_{0} \sin \theta, \\
\hat{\boldsymbol{x}}^{\prime} & =\hat{\boldsymbol{y}}^{\prime} \times \hat{\boldsymbol{z}}^{\prime},
\end{aligned}
$$

where $\theta$ is the angle between $B_{0}$ and $\hat{\boldsymbol{x}}$. Figure 3 shows the magnitude of the flux vector in the rest frame found from Eq. (2). Also shown is the parallel component of the flux vector and the three GSM components of the transverse component of the flux vector. During the period $8-12 \mathrm{~h}$ from the start of the event, it is clear that the transverse component of the flux vector is negligible and that the flux vector is determined only by a component parallel to the magnetic field. The wave is propagated exactly along the magnetic field. As this background field changes, the flux vector remains aligned with it, as discussed by Walker (2014).

Next, we look at the dominant field components $b_{\perp}$ and $v_{\perp}$. The intensities of their GSM coordinates are shown in Fig. 4 . We see that the behaviour of each quantity mirrors that of the other. They are clearly collinear. During the first intensification of wave activity, the direction of polarization rotates from $y$ to $z$ to $x$ directions in the GSM coordinates. This is because the direction of the background field is changing and the wave direction follows it. During the second intensification, the $y$ component dominates.

Consider the ratio $b_{\perp} / \sqrt{\mu_{0} \rho_{0}} v_{\perp}$. This can be calculated from the real part of the analytic signal for each component. Each component varies quasi-sinusoidally so that the phase of this ratio is the phase difference between the two components. Figure 5 show the phase and amplitude of each GSM component of this quantity. During both peaks of wave activity, the phase difference between each component of $b_{\perp}$ 

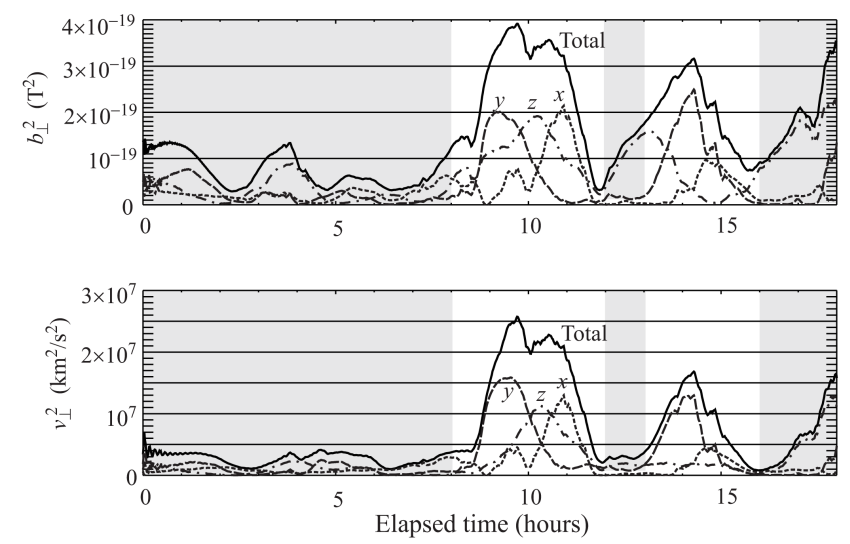

Figure 4. GSM components of $b_{\perp}$ and $v_{\perp}$. Time is measured from 01:31 on 7 June 2000. The unshaded portions of the diagram indicate the peaks in wave energy density.

and $v_{\perp}$ is close to $180^{\circ}$. During the first peak, the amplitude of the ratio is approximately unity. This is not the case for the second peak. It should be noted, however, that the amplitude of the disturbance is smaller during the second interval and the background noise makes a significant contribution to the value. During the first interval, we can deduce that the wave components obey the equipartition relation (Eq. 5), with $p=0$ as for a transverse Alfvén wave, and they are in antiphase as expected.

\section{Conclusions}

The analysis in the previous section leads immediately to the following conclusions about the wave activity in the interval 09:31 to 14:01 UT on 7 June 2000 ( 8 to $12 \mathrm{~h}$ after the start of the event):

- The dominant field perturbations in the wave are the magnetic field and velocity perturbations normal to the ambient magnetic field.

- The wave energy flux vector in the plasma rest frame is parallel to the ambient magnetic field. The wave originates upstream from the solar libration point.

- The transverse components of the magnetic field and velocity are collinear and in antiphase.

- The ratio of the magnitudes of these components is consistent with the theoretical value for a transverse Alfvén wave

All these features are typical of a transverse Alfvén wave. The results are less clear for the second interval from 15:01 to 17:31 but this event is buried deeper in the noise. As determined by Stephenson and Walker (2010), these waves were strongly correlated with a field line resonance observed by the Sanae HF (high-frequency) radar 57 min later.
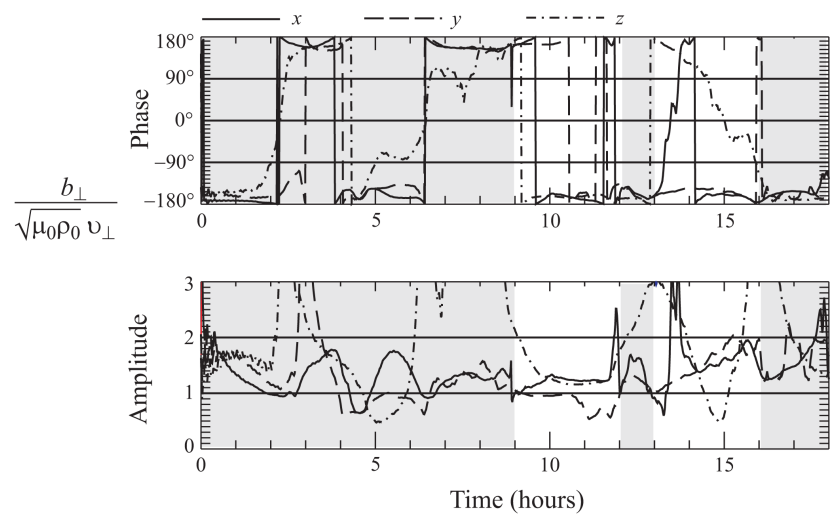

Figure 5. GSM components of the ratio $b_{\perp} / \sqrt{\mu_{0} \rho_{0}} v_{\perp}$. Upper panel shows the phase and the lower shows the amplitude. Time is measured from 01:31 on 7 June 2000. The unshaded portions of the diagram indicate the peaks in wave energy density.

We conclude that during the first interval a transverse Alfvén wave was propagated in the solar wind, travelling downstream past the solar libration point. When it reached the interior of the magnetosphere, it excited a field line resonance at the same frequency. During the second interval a similar process took place, but because it was a weaker event the nature of the MHD (magnetohydrodynamic) wave in the solar wind is less certain.

Acknowledgements. We are grateful to the ACE instrument and science teams of the MAG and SWEPAM instruments for providing the solar wind data, the National Research Foundation of South Africa (NRF) and the South African Department of Environmental Affairs (DEA) for support, and to the members of the SSA-MTM team of the Department of Atmospheric Sciences, UCLA, and the US Geological Survey and the Commissariat à l'Énergie Atomique as well as all other individuals responsible for the development and maintenance of the Toolkit used in the multitaper analysis presented here.

Topical Editor E. Roussos thanks one anonymous referee for his/her help in evaluating this paper.

\section{References}

Ghosh, S., Thomson, D. J., Matthaeus, W. H., and Lanzerotti, L. J.: Coexistence of turbulence and discrete modes in the solar wind, J. Geophys. Res., 114, A08106, doi:10.1029/2009JA014092, 2009.

Stephenson, J. A. E. and Walker, A. D. M.: HF radar observations of Pc5 ULF pulsations driven by the solar wind, Geophys. Res. Lett, 29, 1297, doi:10.1029/2001GL014291, 2002.

Stephenson, J. A. E. and Walker, A. D. M.: Coherence between radar observations of magnetospheric field line resonances and discrete oscillations in the solar wind, Ann. Geophys., 28, 4759, doi:10.5194/angeo-28-47-2010, 2010. 
Thomson, D. J.: Spectrum Estimation and Harmonic Analysis, Proceedings of the IEEE, 70, 1055-1096, 1982.

Walker, A. D. M.: The SHARE radar at Sanae Antarctica, SAJ Science, 98, 257-263, 2002.

Walker, A. D. M.: Magnetohydromagnetic waves in Geospace, IOP Press, Bristol, 2005.
Walker, A. D. M.: Energy exchange and wave action conservation for MHD waves in a general slowly varying medium, Ann. Geophys., submitted, 2014. 